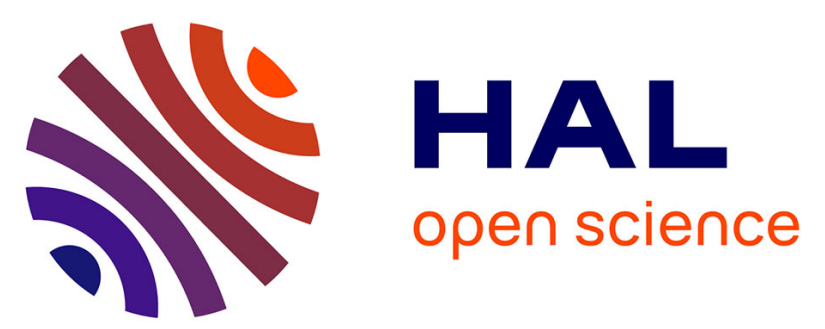

\title{
CONTRIBUTION A L'ÉTUDE DES GROUPES SANGUINS DE LA RACE BOVINE MONTBÉLIARDE. OBSERVATIONS SUR LA STRUCTURE GÉNÉTIQUE DE LA RACE DANS UNE ZONE D'INSÉMINATION ARTIFICIELLE
}

F. Grosclaude, Patrick Millot, P. Ancely, Mireille Faugeras, G. Houlier, Maryvonne Le Duc, G. Vincent

\section{- To cite this version:}

F. Grosclaude, Patrick Millot, P. Ancely, Mireille Faugeras, G. Houlier, et al.. CONTRIBUTION A L'ÉTUDE DES GROUPES SANGUINS DE LA RACE BOVINE MONTBÉLIARDE. OBSERVATIONS SUR LA STRUCTURE GÉNÉTIQUE DE LA RACE DANS UNE ZONE D'INSÉMINATION ARTIFICIELLE. Annales de biologie animale, biochimie, biophysique, 1962, 2 (3), pp.185-208. hal00896174

\author{
HAL Id: hal-00896174 \\ https://hal.science/hal-00896174
}

Submitted on 1 Jan 1962

HAL is a multi-disciplinary open access archive for the deposit and dissemination of scientific research documents, whether they are published or not. The documents may come from teaching and research institutions in France or abroad, or from public or private research centers.
L'archive ouverte pluridisciplinaire HAL, est destinée au dépôt et à la diffusion de documents scientifiques de niveau recherche, publiés ou non, émanant des établissements d'enseignement et de recherche français ou étrangers, des laboratoires publics ou privés. 
Ann. Biol. anim. Bioch. Biophys. I962, 2 (3), I85-208.

\title{
CONTRIBUTION A L'ÉTUDE DES GROUPES SANGUINS DE LA RACE BOVINE MONTBÉLIARDE
}

\author{
OBSERVATIONS SUR IA STRUCTURE GÉNÉTIQUE DE LA RACE \\ DANS UNE ZONE D'INSÉMINATION ARTIFICIELLE \\ F. GROSCLAUDE et P. MILLOT \\ Avec la collaboration technique de P. ANCELy, \\ Mireille Faugeras, G. Houlier, Maryvonne Le Duc et G. Vincent. \\ Laboratoire des Groupes sanguins, \\ Centre national de Recherches zootechniques, Jouy-en-Josas (Seine-et-Oise)
}

\section{SOMMAIRE}

Les groupes sanguins de 400 produits d'un taureau d'insémination artificielle, homozygote pour l'allèle négatif aux loci $\mathrm{B}, \mathrm{J}$ et $\mathrm{Z}$ et possédant un allèle négatif aux loci $\mathrm{A}, \mathrm{S}$ et $\mathrm{L}$ - ainsi que ceux de leurs mères, ont été analysés dans la race bovine Montbéliarde, parente de la race suisse Tachetée Rouge de Simmental.

La série d'anticorps utilisée pour le système $B$, encore incomplète, a permis de distinguer 55 allèles; les plus fréquents sont, hormis l'allèle négatif : $\mathrm{O}_{1} \mathrm{TE}_{\mathbf{3}}^{\prime} \mathrm{I}^{\prime} \mathrm{K}^{\prime}, \mathrm{O}_{\mathbf{1}} \mathrm{TE}_{3}^{\prime} \mathrm{K}^{\prime}$ et $\mathrm{BGKE} \mathrm{K}_{\mathbf{2}}^{\prime} ; p_{i}$ désignant la fréquence de l'un deux, $\Sigma p_{l}^{2}=0,045$; ces résultats indiquent une variabilité génétique relativement forte.

La plupart des allèles semblent identiques à ceux de la race Tachetée Rouge de Simmental (MúlLLER I960). Cependant certains allèles du système $\mathrm{B}$, assez fréquents en race Montbéliarde, ainsi que l'allèle $\mathrm{AZ}^{\prime}$ du système $\mathrm{A}$, n'ont pas été décrits en Suisse. Ėn outre, de fortes différences existent entre certaines fréquences alléliques. Le processus d'isolement génétique des deux races semble donc largement engagé.

L'hypothèse d'équilibre génétique (loi de HARDY-WeinBerg) a été testée aux loci A et SU; elle semble infirmée au locus SU, mais non au locus A. Pourtant une influence non négligeable des premières années d'utilisation de l'insémination artificielle sur les fréquences géniques a été mise en évidence au locus B.

Le sondage effectué définit un état initial de la population, dont l'évolution génétique pourra être contrôlée par des sondages ultérieurs.

\section{INTRODUCTION}

Trois races - ou " rameaux" — se partagent la population bovine française de type Pie Rouge de l'Est, portion la plus occidentale du groupe ethnique Pie Rouge européen; ce sont par ordre d'importance numérique décroissante : la race Mont- 
béliarde, la race Tachetée de l'Est, et la race d'Abondance. Pour ce qui est des deux premières, le rattachement d'un animal à l'une, ou à l'autre, semble assez arbitraire dans certains cas; on constate néanmoins des différences tangibles entre les deux types raciaux, tant dans les caractéristiques "de production " (conformation, aptitudes laitières, rusticité) que dans des caractères sans intérêt économique, tels que l'intensité de la couleur " rouge ".

On possède quelques informations historiques sur la naissance de la race Montbéliarde qui, comme la race Tachetée de l'Est, tire une partie de ses origines de Suisse;

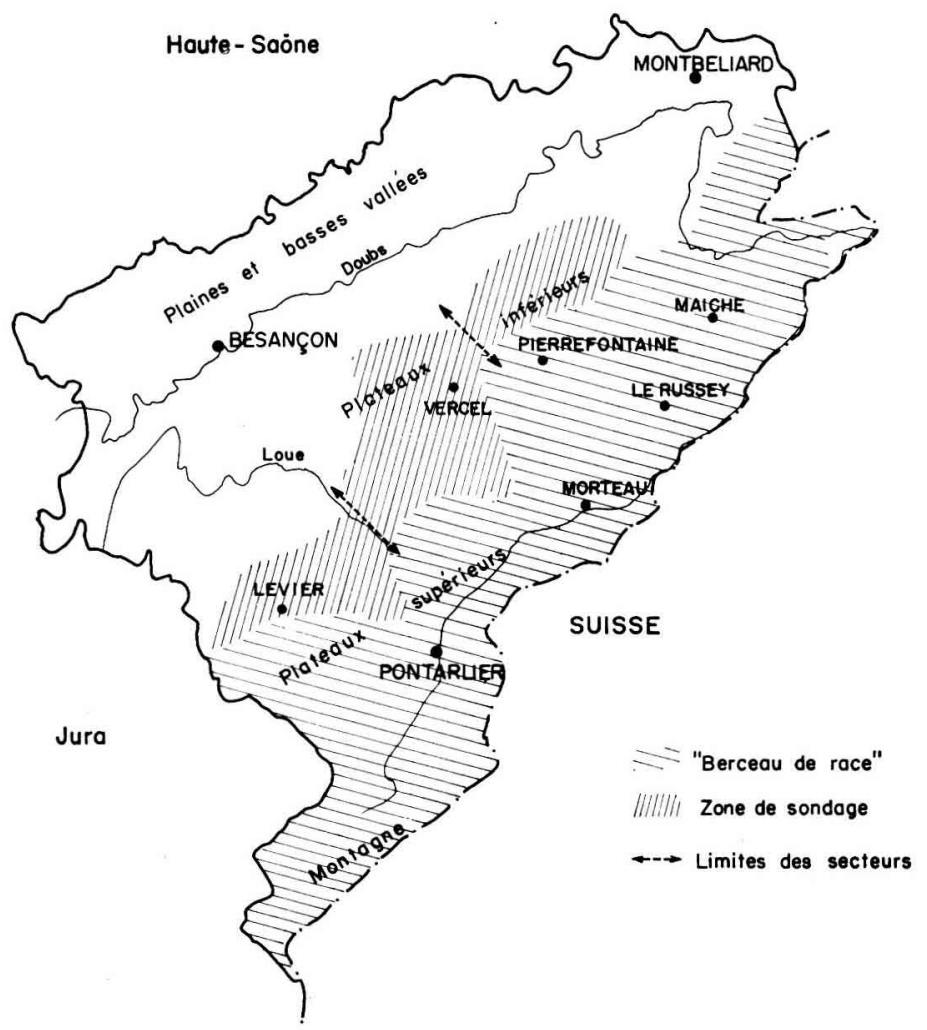

Carte I - Département du Doubs, avec le "herceau "de la race Montbéliarde, où la pratique de l'insémination artificielle est interdite, et la zone de sondage.

mais en race Tachetée de l'Est, 1'immigration de reproducteurs de race Suisse de Simmental reste courante, notamment dans les Centres d'insémination artificielle, alors que les liens de parenté entre le bétail Suisse et la race Montbéliarde sont d'origine plus éloignée.

Selon Vernier (1953), la race Montbéliarde est sour de la race Suisse de Simmental moderne, toutes deux descendant de l'ancien bovin bernois, dont l'ancêtre serait Bos taurus frontosus. Des troupeaux bernois auraient été amenés dans la région de Montbéliard, surtout à partir du début du Xvir ${ }^{\mathrm{e}}$ siècle, par des paysans helvétiques adeptes d'une secte religieuse, qui fuyaient les persécutions. Par ailleurs des importations répétées de bovins suisses eurent lieu également dans la zone frontalière (Franche-Montagne). Peu à peu un type d'animal sélectionné, finalement dénommé 
"Montbéliard ", supplanta les races locales, Fémeline et Tourache. Quoique Vernier affirme que la race Montbéliarde est une pure "bernoise améliorée ", il est vraisemblable qu'il y eut, dans plus d'une étable, croisement d'implantation sur les races locales.

I.es qualités laitières et la rusticité du bovin Montbéliard l'ont fait se multiplier, en premier lieu, dans les départements du Doubs, de la Haute-Saône. puis du Jura, etc., et essaimer dans la zone méditerranéenne et même en Afrique Noire ot̀ il réussit à s'adapter.

La région du Haut-Doubs (carte I) est classée " berceau de la race " et la pratique de l'insémination artificielle y est interdite.

Le présent travail a pour but de décrire les groupes sanguins de la race Montbéliarde, notamment au locus $\mathrm{B}$, et de recueillir ainsi des informations sur la structure génétique actuelle de cette race et sur sa parenté avec la race Suisse de Simmental.

\section{MATÉRIEL ET MÉTHODES}

\section{Io DÉFINITIONS}

A l'exception de l'anti-J qui est un anticorps "naturel ", les anticorps utilisés pour détecter les propriétés antigéniques des hématies des bovins sont obtenus par immunisation (isoimmunisation de bovins, ou hétéroimmunisation de lapins, notamment) et isolés par absorption, selon les techniques mises au point par Ferguson (194I), FERguson, Stormont et Irwin (I942), STORMonT 1950 etc.

Avant d'aborder notre exposé, il est nécessaire de préciser quelques définitions; nous appellerons :

Réactif : un antisérum qui n'est plus fractionnable par absorption; il contient donc en principe un seul type d'anticorps.

Facteur antigénique : le caractère détecté par un réactif. Ces facteurs sont désignés par les lettres de l'alphabet, sans ou avec accent : $A, B, C \ldots Z, A^{\prime}, B^{\prime} \ldots Z^{\prime}$.

Phénogroupe: un groupe de facteurs commandé par un gène; on utilisera également le terme d'antigène, ou celui d'allèle.

Système : la série allélique correspondant à un locus.

Groupe sanguin : la liste des facteurs antigéniques (phénotype sanguin), ou des allèles aux différents systèmes (génotype sanguin), que possède un animal.

\section{$2^{\circ}$ RÉACTIFS UTILISÉ́S}

Les anticorps hémolytiques utilisés dans cette étude (tableau I ci-dessous) ont été préparés depuis 1956 par l'un de nous (MilLOT) sauf anti-K, anti-K' et anti-L', fournis par Y. BouQUET (Laboratoire de Gand, Belgique). Les anticorps anti- $\mathrm{H}$, anti- $\mathrm{J}^{\prime}$ et ant $\mathrm{i}^{-} \mathrm{D}^{\prime}$, donnés par les laboratoires de Copenhague (Danemark) et de Munich (Allemagne), n'ont été utilisés que pour quelques dizaines d'analyses, ainsi que l'anti-P et l'anti- $\mathrm{Y}^{\prime}$,'récemment obtenus par nous-mêmes. Anti-N $f$ et anti- $0^{\prime \prime}$ sont deux réactifs correspondant à des facteurs antigéniques du système B qui semblent n'avoir été obtenus que dans notre laboratoire.

TABIEAU 2

Liste par locus des réactifs utilisés

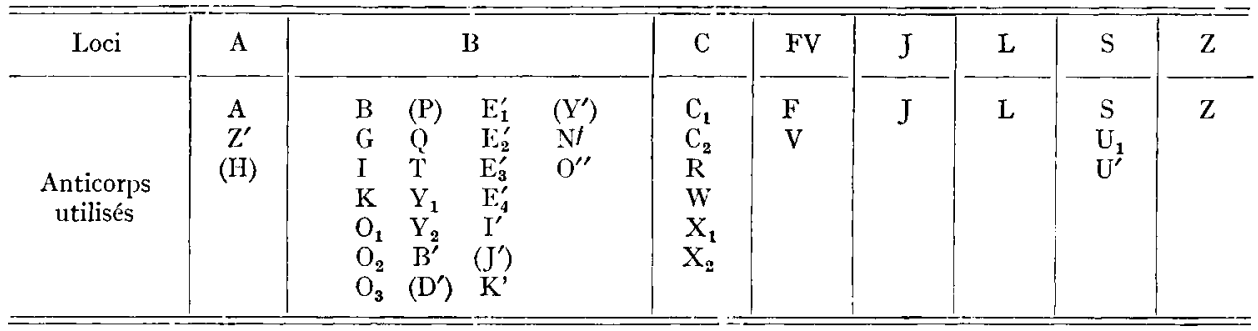

Les parenthèses indiquent les réactifs qui n'ont été utilisís que pour une partie des analyses. 
Depuis les tests de comparaison internationaux de $196 \mathrm{r}$, plusieurs améliorations sont intervenues dans cette série d'anticorps:

Les réactifs anti- $Q_{1}$, anti-I' et anti-C $C_{1}$ ont été renouvelés et sont supérieurs aux réactifs antérieurs; la première moitié des couples de l'échantillon n'ayant pas été examinée avec le nouvel anti-I', le sang de la plupart des produits fut prélevé et analysé une seconde fois. Ce sont ces analyses qui ont bénéficié par ailleurs des anticorps $\mathrm{P}, \mathrm{D}^{\prime}, \mathrm{J}^{\prime}, \mathrm{Y}^{\prime}$, ainsi que d'un nouvel anti- $\mathrm{O}_{3}$ supérieur au précédent, qui ne réagissait pas avec certains globules possédant le caractère $\mathrm{O}_{3}$.

En ce qui concerne l'anti-B, l'interprétation des résultats s'est trouvée améliorée quand nous eûmes établi que le sérum utilisé était en réalité anti-B $+\mathrm{H}$ : néanmoins l'anti-B du mélange est un peu faible et ne décèle pas le facteur antigénique régulièrement dans tous les phénogroupes qui le comportent ; cette irrégularité est liée aux variations qualitatives du complément.

Par ailleurs nous avons tenu compte du fait que l'anti- $\mathrm{E}_{3}^{\prime}$ et l'anti-Z contenaient également l'anticorps anti-Z'.

\section{$3^{\circ}$ Technique D'ANAlyse}

On distribue dans des tubes d'hémolyse deux gouttes (environ o, $10 \mathrm{cc}$ ) de réactif, puis une goutte de suspension à I p. Ioo dans l'eau physiologique (eau distillée + 9, I $2 \mathrm{~g}$ par litre de $\mathrm{NaCl}$ ) d'hématies lavées à trois reprises dans l'eau physiologique; les tubes sont fortement agités; après I $5 \mathrm{mn}$ on ajoute une goutte de complément, sous forme de sérum de lapin enrichi de $1 / 10$ de sérum de cobaye. L'activité complémentaire de ce mélange est plus constante que celle du sérum de lapin pur. Les tubes sont réagités et maintenus en chambre isotherme à $24^{-2} 6^{\circ} \mathrm{C}$.

Trois lectures visuelles du degré d'hémolyse sont effectuées respectivement une heure, trois heures et quatre heures après la distribution du complément, les tubes étant agités lors des lectures; le degré d'hémolyse est noté selon une échelle linéaire : \pm (traces), I, 2, 3, 4 (hémolyse totale); chaque échantillon de sang est analysé deux fois, en principe par deux opérateurs différents ; les réactions donnant des résultats divergents sont refaites, en double également.

\section{a) Aspect théorique}

\section{$4^{0}$ MÉthode}

L'objectif principal de cette étude était la description et le calcul de la fréquence des phénogroupes allèles du locus B. Deux méthodes étaient suggérées par les travaux d'autres auteurs :

Méthode directe.

On forme un échantillon de taureaux d'effectif $\mathrm{N}$, représentatif de la race, et on détermine les deux phénogroupes que chacun d'eux possède ; cette détermination s'effectue à l'aide de couples mère-produit dans la descendance du taureau. En moyenne 5 à 6 couples sont nécessaires, donc 5 à 6 analyses pour connaître un phénogroupe.

\section{Méthode de NeImann-Sorensen (I956) :}

Sa justification mathématjque a été fournie par CEPPELlini et al. (I956). Cette méthode comporte deux phases : dans une première phase, l'examen systématique des groupes sanguins de familles (couples mère-produit dans la descendance d'un taureau, par exemple) permet de reconnaître les phénogroupes les plus fréquents. Dans une seconde phase, la fréquence des phénogroupes découverts est estimée par un processus itératif dont l'application suppose, en toute rigueur, l'équilibre génétique réalisé (loi de HARDY-WEINBERG). Cette méthode est un peu moins coûteuse en analyses, pour un même volume d'informations, que la méthode précédente. Mais Ie fait d'admettre à priori qu'il y a équilibre génétique est certainement inexact dans beaucoup de populations bovines actuelles, et empêche précisément les observations sur leur structure génétique réelle. Aussi avons-nous été amenés à essayer une autre méthode.

Méthode utilisée : elle consiste à utiliser la descendance d'un taureau d'insémination artificielle qui soit homozygote pour l'allèle négatif au locus B (le premier visé par l'étude) et qui possède aux autres loci un maximum d'allèles négatifs. L'utilisation de ce taureau réalise, pour les loci où il est homozygote négatif, un vaste test-cross dans la population des femelles.

Cette méthode qui n'implique aucune hypothèse génétique préalable est particulièrement économique en analyses : le phénotype de chaque descendant du taureau est un allèle tiré, - au hasard sous certaines conditions, - dans la population des mères.

Il suffit donc, parmi les mères croisées avec le taureau homozygote négatif, de construire un échantillonnage représentatif de la population considérée, qui ne comprend, naturellement, que des élevages pratiquant l'insémination artificielle. Le choix de l'effectif de l'échantillon dépend de la précision désirée pour l'estimation des fréquences; quelle que soit d'ailleurs la méthode utilisée, il s'agit d'un modèle multinominal dans lequel la précision des estimations n'est satisfaisante qu'à partir de grands échantillons. 
Remarquons qu'en réalité “ l'allèle négatif " représente le groupe d'allèles ne réagissant avec aucun des réactifs mis au point jusqu'ici. Avec la série de réactifs utilisée dans ce travail, sa fréquence reste élevée, dans toute les races sans doute; il y existe donc toujours un certain nombre de taureaux homozygotes négatifs. Ultérieurement la mise au point de réactifs supplémentaires doit permettre de compléter et diversifier la série allélique mise en évidence par test-cross.

\section{b) Réalisation pratique}

Les conditions voulues étaient remplies dans le département du Doubs par le taureau Élégant HBM I 3.748 dont le phénotype est, avec les anticorps utilisés :

et dont le génotype s'est avéré être :

$$
\mathrm{A} /-\left|\mathrm{C}_{2} \mathrm{X}_{2} \mathrm{~L}^{\prime} / \mathrm{F}\right|-\mid \mathrm{L} / \mathrm{S} /-1
$$

$$
\frac{\mathrm{A}}{-} /=\left|\frac{\mathrm{C}_{2} \mathrm{X}_{2} \mathrm{~L}^{\prime}}{\mathrm{C}_{2}}\right| \frac{\mathrm{F}}{\mathrm{F}}|=| \frac{\mathrm{L}}{-}\left|\frac{\mathrm{S}}{-}\right| \frac{-}{-} \mid
$$

L'application stricte de la méthode des sondages aléatoires, avec tirage au sort des unités de l'échantillon parmi toutes les unités de la population, préalablement repérées, présentant de grandes difficultés matérielles, il a été procédé de la façon suivante :

- l'effectif de l'échantillon a été fixé à 400 couples mère-produit,

- tous les couples existant dans les élevages, affiliés ou non au Livre Généalogique, où la paternité du taureau pouvait être admise avec vraisemblance ont été retenus, et ceci dans une zone bordant le berceau de la race ; cette zone s'est alors étendue progressivement jusqu'à la réunion des 400 couples désirés (carte $\mathrm{I}$ ),

- les prélèvements de sang ont été effectués du 28 décembre ig60 au 26 mai i95i dans 59 communes et 252 élevages; dans chaque élevage étaient en outre recensés: le nombre de vaches exploitées, le nombre d'ent re elles issues d'insémination artificielle, ainsi que le nom du père de chaque mère retenue dans l'échantillon, s'il était un taureau d'insémination artificielle.

\section{c) Observations sur les caractéristiques du sondage}

Dans la zone de sondage coexistent les élevages utilisant et n'utilisant pas l'insémination artificielle. Les premiers, seuls visités, groupent la plus grosse partie de l'effectif, les deux tiers au moins semble-t-il. Par ailleurs il est difficile de préciser objectivement la limite séparant les élevages admis à être représentés dans l'échantillon de ceux qui n'ont pas été retenus en raison de leur niveau technique insuffisant; des élevages parfois médiocres ont fourni des couples mère-produit, et on pourra contester qu'ils puissent représenter la race Montbéliarde. En fait la zone de sondage est un vieux fief de cette race et une telle objection ne semble pas fondée.

Mais l'échantillon n'est réellement représentatif de la population ainsi grossièrement délimitée que si certaines conditions sont remplies, et en particulier :

- Aucune sélection gamétique $\left(^{1}\right)$ chez les mères, ce qui est vraisemblable, compte tenu des résultats acquis chez les bovins par les auteurs étrangers,

- Aucune sélection zygotique dans la descendance du taureau, ce qui semble avoir été le cas ; les inséminations sont faites sensiblement au hasard, et Élégant r3.748 ayant bonne réputation, tous ses veaux femelles sont en principe élevés,

- Taux de sondage indépendant de l'effectif $\mathrm{N}$ des élevages; les chiffres obtenus, préalablement traités par la méthode des moyennes mobiles (en groupant pour l'effectif $\mathrm{N}$ les élevages d'effectif recensé $: N \longrightarrow I, N, N+I)$ sont les suivants :

\section{TABIEAU 2}

Taux de sondage en fonction de l'effectif des élevages

\begin{tabular}{rl|c|c|c|c|c|c|c|c|c|c|c|c|c|c}
\hline $\begin{array}{c}\text { Effectif N des élevages } \\
\text { (vaches laitières) .... }\end{array}$ & 6 & 7 & 8 & 9 & 10 & 11 & 12 & 13 & 14 & 15 & 16 & 17 & 18 & 19 & 20 \\
\hline $\begin{array}{c}\text { Nombre d'élevages ..... } \\
(\mathrm{N}-\mathrm{I})+\mathrm{N}+\mathrm{N}+\mathrm{I})\end{array}$ & 14 & 23 & 45 & 69 & 73 & 88 & 70 & 69 & 49 & 47 & 36 & 23 & 14 & 16 & 18 \\
\hline \begin{tabular}{c} 
Taux de sondage apparent \\
\hline
\end{tabular} & $-18,6$ & 19,1 & 17,8 & 16,0 & 14,4 & 13,6 & 12,7 & 12,8 & 11,0 & 11,3 & 10,8 & 10,6 & 11,7 & 10,5 & 9,9 \\
\hline
\end{tabular}

( ${ }^{1}$ On entend par sélection gamétique. une sélection s'opérant sur les gamètes dont certains, porteurs de gènes ou combinaisons géniques favorisées. sont plus souvent fécondants que d'autres. 
Contrairement aux apparences, ces chiffres indiquent que le taux de sondage réel a été sensiblement indépendant de l'effectif des élevages et voisin de 8 p. 10o,

Soient $t_{i}$ le taux de sondage apparent dans les élevages d'effectif $n_{i}$

$\mathrm{N}_{i}$ le nombre d'élevages d'effectif $n_{\imath}$

On a:

$p_{i}$ le taux de sondage réel dans les élevages d'effectif $n_{i}$

$$
t_{i}=\frac{\text { nombre de vaches retenues }}{\text { nombre total de vaches dans les élevages ayant fourni des couples }} .
$$

La probabilité qu'un élevage fournisse un couple au moins est :

donc

$$
\begin{gathered}
\mathrm{I}-\left(\mathrm{I}-p_{i}\right)^{n_{i}} \\
t_{i}=\frac{p_{i} n_{i} \mathrm{~N}}{n_{i} \mathrm{~N}_{i}\left[\mathrm{I}-\left(\mathrm{I}-p_{i}\right)^{n}\right]}=\frac{p_{i}}{\mathrm{I}-\left(\mathrm{I}-p_{i}\right)^{n}} .
\end{gathered}
$$

Si $p$ est indépendant de $n$, donc constant, les valeurs obtenues pour les différentes valeurs de $n$ se trouvent sur la courbe d'équation

$$
t=\frac{p}{\mathrm{I}-(\mathrm{I}-p)^{n}} .
$$

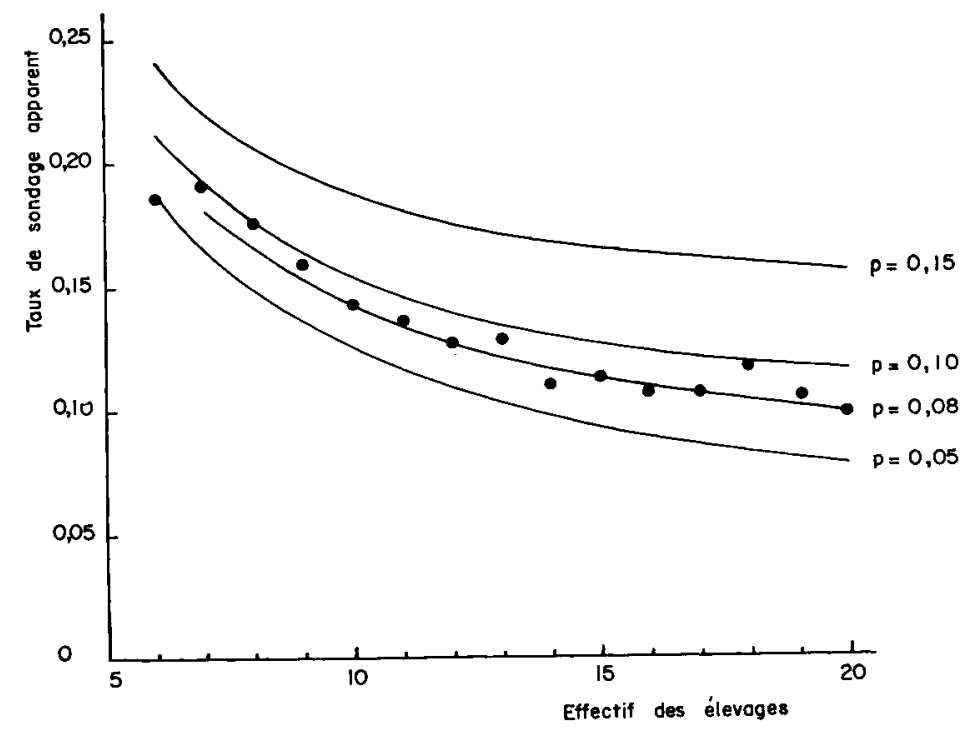

Fig. I - Taux de sondage apparent en fonction du taux de sondage réel $: \mathrm{p}$ et de l'etfectif des élevages. Les valeurs sont données sous tarme de courbes continues pour faciliter la lecture. Les points indiquent les taux de sondage apparents oblenus dans l'échantillonnage. La courbe : $\mathrm{p}=0,08$ correspond à leur meilleur aịstement ( $\chi_{2}$ minimum).

La figure I montre que cette condition est sensiblement remplie. Le meilleur ajustement, correspondant au $\chi^{2}$ minimum, est obtenu pour $p=0,08$.

- Homogénéité du sondage dans l'espace. Pour diverses considérations, nous avons distingué 3 secteurs dans la zone de sondage (carte I) où la structure de la population bovine aurait pu présenter de légères différences. Le tableau 3 donne quelques caractéristiques concernant ces secteurs.

L'insémination artificielle s'est développée moins vite dans le secteur nord que dans les deux autres, mais actuellement, les différences sont moins accusées que ne l'indique la ligne 2, qui reflète la situation existant il y a quelques années. Le taux de sondage apparent croît légèrement du nord au sud; en fait, compte tenu de l'effectif moyen des élevages, les taux réels de sondage pour les secteurs centre et sud sont équivalents; la région nord est légèrement sous-représentée. Il est impossible de 
définir le sondage par rapport à la population totale des secteurs, les statistiques agricoles étant très infidèles; néanmoins on peut estimer que les effectifs de la ligne $\mathrm{I}$ représentent environ $\mathrm{I} / 6$ à I/7 de l'effectif total du secteur.

TABLEAU 3

Caractéristiques de la zone de sondage subdivisée en secteurs

\begin{tabular}{|c|c|c|c|c|}
\hline & \multicolumn{3}{|c|}{ Secteurs } & \multirow{2}{*}{ Totaux } \\
\hline & Nord & Centre & Sud & \\
\hline $\begin{array}{r}\text { Effectif des vaches laitières dans les } \\
\text { élevages visités } \ldots \ldots \ldots \ldots \ldots \ldots \ldots\end{array}$ & 1048 & 1222 & 853 & 3123 \\
\hline $\begin{array}{l}\text { Pourcentage de vaches issues d'insé- } \\
\text { mination artificielle dans ce secteur... }\end{array}$ & 40,6 & 55,8 & 52,1 & 49,7 \\
\hline Nombre de couples retenus .......... & 119 & 158 & 123 & 400 \\
\hline Taux de sondage apparent........ & 11,4 & 12,9 & $1: t, \dot{4}$ & 12,8 \\
\hline $\begin{array}{l}\text { Effectif moyen des élevages repré- } \\
\text { sentés dans l'échantillon ........... }\end{array}$ & 12,3 & 13,7 & 11,1 & 12,4 \\
\hline
\end{tabular}

\section{RÉSULTATS}

\section{Io Locus A}

Les trois méthodes de calcul des fréquences géniques utilisées dans ce travail vont être développées dans le cas du Locus A, ainsi que le principe du test de l'hypothèse d'équilibre génétique.

\section{a) Méthodes de calcul des fréquences géniques}

\section{Calcul par comptage chez les produits :}

Cette méthode s'applique quand il y a test-cross, donc dans le cas de l'allèle $A Z$ ' [STORMON'r (I96I) signale que le facteur $Z^{\prime}$ appartient au système $A$; effectivement dans 209 phénotypes d'animaux Charolais, Normands et Limousins, $Z$ ' est toujours associé à $\mathrm{A}$, et, dans les filiations étudiées, il est transmis par l'allèle $\left.\mathrm{A} Z^{\prime}\right]$. La fréquence de l'allèle $A Z^{\prime}$ est donc estimée par celle du facteur $Z^{\prime}$ chez les produits. Les chiffres du tableau 4 conduisent à l'estimation :

$$
f_{A Z^{\prime}}=0,0 \mathrm{I} 7 \pm 0,0 \mathrm{O} 3
$$

Cette fréquence étant faible, son estimation est relativement imprécise.

Calcul par la méthode dite "de la racine carrée".

Dans 1'hypothèse d'équilibre génétique, la fréquence $f_{a}$ de l'allèle négatif $a$ peut être estimée par : $f_{a}=\sqrt{\frac{\alpha}{\mathrm{N}}}$, où $\alpha$ est le nombre de mètres de phénotype $[a]$. 
L'écart type de cette estimation est $s_{/ a}=\sqrt{\frac{\overline{I-f_{a}^{2}}}{4 \mathrm{~N}}}$.

On obtient ici, d'après le tableau 4 :

$$
\begin{gathered}
f_{a}=0,557 \pm 0,042 \\
\text { TABLEAU } 4 \\
\text { Analyse du locus } A
\end{gathered}
$$

\begin{tabular}{|c|c|c|c|c|c|c|}
\hline Phénotype de la mère & A & A & $a$ & $a$ & $Z^{\prime}$ & $Z^{\prime}$ \\
\hline Phénotype du produit ....... & $\AA$ & $a$ & $a$ & A & $Z^{\prime}$ & \\
\hline \multirow[t]{2}{*}{ Nombre de cas observés ..... } & 228 & 48 & 59 & 65 & 7 & 7 \\
\hline & & $\beta$ & & & & \\
\hline
\end{tabular}

La ségrégation observée pour le facteur $Z^{\prime}$ est présentée indépendamment, par commodité.

Calcul par comptage chez les mères :

La fréquence de l'allèle $a$ peut être estimée sans faire appel à 1'hypothèse d'équilibre génétique en utilisant:

- le nombre de mères de phénotype $[a]$, donc de génotype $a / a$

- le nombre de produits de phénotype $[a]$ issus des mères de phénotype $[A]$ : ces produits sont issus de mères de génotype $\mathrm{A} / a$; ils apparaissent avec la probabilité I/ 4 puisque leur père a le génotype $\mathrm{A} / a$.

L'estimation de $f_{a}$ par la méthode du maximum de vraisemblance est alors :

$$
f_{a}^{\prime}=\frac{\alpha+2 \beta}{\mathrm{N}} \text {. }
$$

I'écart type de cette estimation est donné par :

$$
s f_{a}=\sqrt{\frac{\alpha(\mathrm{N}-\alpha)+4 \beta(\mathrm{N}-\alpha-\beta)}{\mathrm{N}^{3}}} .
$$

Les valeurs du tableau 4 conduisent à l'estimation :

$$
f_{a}=0,550 \pm 0,070 \text {. }
$$

Cette valeur n'est pas significativement différente de celle trouvée par la méthode de la racine carrée $(t=0, \mathrm{I} 7)$.

\section{b) Test de l'hypothèse d'équilibre génétique}

Pour tester l'hypothèse d'équilibre génétique, il suffit de comparer, par un test de $\chi^{2}$ (I degré de liberté) le groupe des valeurs observées :

$$
\alpha, \quad \beta, \quad N-\alpha-\beta
$$

au groupe des valeurs théoriques correspondantes, calculées dans l'hypothèse d'équilibre génétique :

$$
\mathrm{N} f_{a}^{2}, \quad \frac{\mathrm{N} f_{a}\left(\mathrm{I}-f_{a}\right)}{2}, \quad \mathrm{~N}-\frac{\mathrm{N} f_{a}\left(\mathrm{I}+f_{a}\right)}{2} .
$$


Les chiffres obtenus sont les suivants :

$\begin{array}{llll}\text { Valeurs observées : } & \text { I } 24 & 48 & 228 \\ \text { Valeurs théoriques : } & \text { I } 2 \text { I } & 49,5 & 229,5\end{array}$

On trouve $\chi^{2}=0$, I3. I'hypothèse d'équilibre génétique n'est donc pas infirmée.

\section{$2^{\circ}$ Locus S}

En admettant qu'en race Montbéliarde comme dans les races américaines (STORmont, I95I), les phénogroupes $\mathrm{SU}_{1}, \mathrm{SU}^{\prime}$ et $\mathrm{U}_{1} \mathrm{U}^{\prime}$ n'existent pas, l'emploi des anticorps $S, U_{1}$ et $U^{\prime}$ permet de distinguer les 4 allèles $S, U_{1}, U^{\prime}$, et s. Leur fréquence est estimée à partir des chiffres du tableau 5 , et en tenant compte du génotype $S / \mathrm{s} d u$ taureau Élégant.

\section{TABLEAU 5}

Analyse du locus SU

\begin{tabular}{|c|c|c|c|c|c|c|c|c|}
\hline$\frac{\text { Phénotype de la mère }{ }^{(\mathbf{1})}}{\text { Phénotype du produit }{ }^{(1)}}$ & $\begin{array}{l}\mathrm{S} \\
\downarrow \\
\mathrm{S}\end{array}$ & $\begin{array}{l}\mathrm{S} \\
\downarrow \\
s\end{array}$ & $\begin{array}{l}s \\
\downarrow \\
s\end{array}$ & $\stackrel{s}{\downarrow}$ & $\begin{array}{l}\mathrm{U}_{1} \\
\downarrow \\
\mathrm{U}_{1}\end{array}$ & $\begin{array}{l}\mathrm{U}_{1} \\
\downarrow \\
u\end{array}$ & $\begin{array}{c}\mathbf{U}^{\prime} \\
\downarrow \\
\mathrm{U}^{\prime}\end{array}$ & $\begin{array}{l}\mathrm{U}^{\prime} \\
\downarrow \\
u^{\prime}\end{array}$ \\
\hline Nombre de cas observés & 134 & 46 & 120 & 100 & 9 & 12 & 12 & 20 \\
\hline
\end{tabular}

(1) Pour l'allèle considéré seulement.

Pour $f_{s+\mathrm{U}_{1}+\mathrm{U}^{\prime}}$, on obtient : $0,780 \pm 0,064$ par la méthode du comptage chez les mères et $0,740 \pm 0,035$ par la méthode de la racine carrée. Ces deux estimations ne sont pas significativement différentes $(t=r, 08)$.

Le test de l'hypothèse d'équilibre génétique s'opère sur les valeurs suivantes :

$$
\begin{array}{llll} 
& \multicolumn{1}{c}{\alpha,} & \beta, & \mathrm{N}-\alpha-\beta . \\
\text { Valeurs théoriques } & 243,36 & 34,32 & \text { I22,32 } \\
\text { Valeurs observées } & 220 & 46 & \text { I34 }
\end{array}
$$

On trouve : $\chi^{2}=7,33$. L'hypothèse est donc infirmée. Selon ces chiffres la déviation se manifesterait par un excès d'hétérozygotes et d'homozygotes S/S. Dans un groupe de 128 mères issues d'insémination artificielle, le nombre des mères de phénotype (S) est de 66 , donc 5I p. Ioo contre 45 p. Ioo dans l'échantillon qui englobe d'ailleurs ce groupe. Il est donc possible que la fréquence du gène $S$ chez les taureaux d'insémination artificielle ait été supérieure à celle de la population. Néanmoins la déviation observée pourrait également être purement aléatoire.

Les fréquences $f_{\mathrm{U}_{1}}$ et $f_{\mathrm{U}^{\prime}}$, sont estimées par comptage chez les produits. Finalement on obtient :

$$
\begin{aligned}
& f_{\mathrm{s}}=0,220 \quad \pm 0,064 \\
& f_{\mathrm{U}_{1}}=0,023 \quad \pm 0,015 \\
& f_{\mathrm{U}^{\prime}}=0,030 \quad \pm 0,017 \\
& f_{s}=0,727 \quad \pm 0,069
\end{aligned}
$$


Les deux fréquences $f_{\mathrm{v}_{1}}$ et $f_{\mathrm{v}^{\prime}}$, étant faibles, on peut aussi les estimer chez les mères en admettant 1 'absence des homozygotes, ce qui donne $t_{\mathrm{V}_{1}}=0,026 \pm 0$,o I I et $f_{\mathrm{u}^{\prime}}=0,040 \pm 0,0 \mathrm{I}_{4}$

\section{$3^{\circ}$ Locus FV}

Le locus FV est le locus qui se prête le mieux au test de l'hypothèse d'équilibre génétique puisque la connaissance des génotypes est immédiate. En fait l'anti-F utilisé étant faible, le nombre de 6 homozygotes $\mathrm{V} / \mathrm{V}$ observés n'est pas certainement exact; dans ces conditions, l'hypothèse d'équilibre génétique ne peut être valablement vérifiée à ce locus. Les chiffres observés, donnés par le tableau 6 , conduisent à l'estimation :

$$
f_{v}=\frac{n+2 m}{2 \mathrm{~N}}=0,08 \pm 0,02
$$

TABLEAU 6

Analyse du locus FV

\begin{tabular}{|c|c|c|c|}
\hline Génotype de la mère ..... & $\mathrm{V} / \mathrm{V}$ & $\mathrm{V} / \mathrm{F}$ & $\mathrm{F} / \mathrm{F}$ \\
\hline \multirow{2}{*}{ Nombre de cas observés ... } & 6 & 52 & 342 \\
\hline & $m$ & $n$ & \\
\hline
\end{tabular}

$4^{\circ}$ Locus J

Dans la descendance de 128 mères possédant l'antigène $\mathrm{J}$ absent chez le père 53 produits seulement possèdent cet antigène, ce qui s'explique par la présence dans l'échantillon de veaux très jeunes (on sait en effet que l'antigène $J$ n'est détectable que quelques semaines après la naissance). La meilleure estimation de la fréquence de $\mathrm{J}$ est alors le complément de celle de $j$, obtenue par la méthode de la racine carrée, soit :

$$
f_{s}=0,175 \pm 0,028
$$

\section{$5^{\circ}$ Locus L}

Les résultats obtenus au locus $\mathrm{L}$ présentent une anomalie. En effet, dans la descendance des mères de phénotype $[l]$, le gène $\mathrm{L}$, du taureau Élégant est transmis moins souvent que son allèle $l$. Cette différence est statistiquement significative au seuil 5 p. IOO.

TABLEAU 7

Analyse du locus $L$

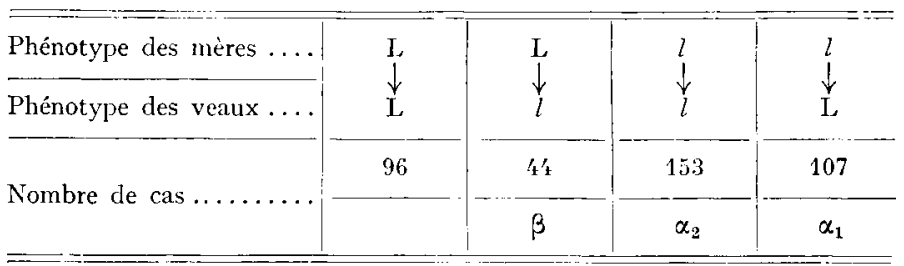


Il ne semble pas qu'il y ait eu ségrégation anormale du couple de gènes du taureau : les proportions observées s'expliquent sans doute par un défaut d'affinité de l'anti-L pour certains globules de jeunes animaux, phénomène que nous avons pu observer également dans le cas d'un autre anticorps, non utilisé dans cette étude.

L'estimation de $f /$ par la méthode de la racine carrée a pour valeur : $0,806 \pm 0,030$ donc $f_{\mathrm{L}}=0, \mathrm{I} 94 \pm 0,030$. L'estimation ponctuelle par comptage chez les mères est : $f_{l}=0,86$. En fait la valeur de $\beta$ est sans doute faussée par la " ségrégation anormale "; il est préférable d'estimer le nombre de mères hétérozygotes par l'expression :

$$
\frac{2\left(\alpha_{1}+\alpha_{2}\right) \beta}{\alpha_{2}} \text { au lieu de } 4 \beta \text {. }
$$

On obtient alors $h_{l}=0,837$, estimation qui se rapproche de celle obtenue cidessus par la méthode de la racine carrée.

\section{$6^{\circ}$ Locus $Z$}

Le sérum anti- $Z$ contenant également anti- $Z^{\prime}$, les résultats (tableau 8 ) portent sur 386 couples après élimination de I4 couples où la mère est $Z^{\prime}$.

\section{TABLEAU 8}

Analyse du locus $Z$

\begin{tabular}{l|c|c|c}
\hline Phénotype de la mére ... & $\begin{array}{c}Z \\
\downarrow \\
\text { Phénotype du produit ... }\end{array}$ & $\begin{array}{c}\mathrm{J} \\
\mathrm{Z}\end{array}$ & $\begin{array}{c}\mathrm{z} \\
\mathrm{z}\end{array}$ \\
\hline Nombre de cas observés... & 103 & 125 & 158 \\
\hline
\end{tabular}

Par comptage chez les produits, on obtient $f_{\mathrm{z}}=0,267 \pm 0,045 ;$ par comptage chez les mères $f_{z}=\frac{\alpha+\beta}{N}=0,733 \pm 0,045$, valeurs qui concordent exactement. Mais la méthode de la racine carrée donne $: f_{z}=0,640+0,036$, qui diffère significativement de la valeur précédente $(t=3,23)$. L'hypothèse d'équilibre génétique serait infirmée $\left(\chi^{2}=44,14\right)$. Ceci pourrait être dû à un excès d'hétérozygotes imputable aux taureaux d'insémination artificielle; cela ne semble pas être le cas puisque dans un groupe de $I 24$ filles de ces taureaux, la fréquence du phénotype ( $Z$ ) est de 0,69 I contre $0,68 \mathrm{I}$ dans l'échantillon et la fréquence des mères ne transmettant pas $Z$ à leur produit $=0,323$ contre 0,324 dans l'échantillon. En fait, comme l'anti-L, l'anti-Z n'est pas entièrement satisfaisant, ce qui explique vraisemblablement cette différence.

\section{$7^{\circ}$ Locus C}

Le locus $\mathrm{C}$ est le seul locus où le génotype du taureau Élégant se prête mal à la détermination des phénogroupes; en principe tout phénogroupe d'au moins deux facteurs comportant notamment $C_{2}$ ne peut être déterminé ; mais outre $C_{2} X_{2} L^{\prime}$ 
figurant chez le taureau, l'existence de $\mathrm{C}_{2} \mathrm{~W}$ dont $\mathrm{C}_{2}$ est plus fort dans la race que celui de l'allèle $\mathrm{C}_{2}$ du taureau est très vraisemblable.

Par ailleurs, ont été reconnus les allèles : $c, \mathrm{C}_{2}, \mathrm{C}_{1}, \mathrm{~W}, \mathrm{~L}^{\prime}$ et $\mathrm{C}_{1} \mathrm{~W}$. De façon générale la fréquence des facteurs $C_{2}, W, X_{2}$ et $L^{\prime}$ est très élevée dans la race. L'étude du locus C sera poursuivie.

\section{$8^{\circ}$ Locus B}

\section{a) Description et fréquence des allèles}

54 allèles différents ont été reconnus au locus B chez les 400 produits (tableau 9). Mais certains phénogroupes maternels ne s'expliquent pas par les allèles cités; un $55^{\mathrm{e}}$ allèle, non transmis aux produits, a été reconnu chez les mères: $\mathrm{GQY}_{2} \mathrm{~B}^{\prime} \mathrm{O}^{\prime \prime} \mathrm{Y}^{\prime} \mathrm{N}_{f}$, un $5^{6^{\mathrm{e}}}$ est possible : $\mathrm{BGKB}^{\prime} \mathrm{E}_{2}^{\prime} \mathrm{I}^{\prime}$. En outre, il est vraisemblable que certains allèles de la race ne sont pas représentés dans l'échantillon. La fréquence à partir de laquelle un gène rare a $95 \mathrm{p}$. roo de chances de ne pas apparaître dans un échantillon de 400 individus est, suivant le modèle de PoIsson, de $125 \cdot \mathrm{IO}^{-6}$. Mais de toute façon il est inévitable que certains allèles, même moins rares, ne figurent pas dans l'échantillon. L'examen des intervalles de confiance donnés à titre indicatif pour chaque fréquence considérée indépendamment des autres, rappelle d'ailleurs l'imprécision d'une estimation calculée à partir d'un échantillon de 400 unités seulement.

Nos résultats ne sont vraisemblablement pas exempts d'erreurs; celles-ci peuvent provenir de deux sources :

a) Une erreur de paternité non décelée. - Dans ce cas le groupe defacteurs retenu comme phénogroupe représente en réalité 2 phénogroupes superposés ; de tels phénogroupes erronés, s'il en est, doivent figurer parmi les phénogroupes les plus rares. Pour limiter ce type d'erreur, toute filiation tant soit pet1 douteuse a été exclue; de toute façon plusieurs phénogroupes qui ne sont apparus qu'une seule fois dans l'échantillon ont pu être rencontrés par ailleurs : $\mathrm{BGKQB} \mathrm{E}_{2}^{\prime} \mathrm{O}^{\prime \prime}, \mathrm{BQN}_{f}, \mathrm{BO}_{1}, \mathrm{~B}^{\prime} \mathrm{O}^{\prime \prime}$; leur existence est donc confirmée.

b) Certains défauts des réactifs utilisés. - De grandes précautions ont été prises en ce qui concerne les réactifs que nous savions imparfaits. Néanmoins quelques doutes subsistent dans certains cas ; ainsi $\mathrm{O}^{\prime \prime}$ présentant parfois des réactions assez faibles, donc sensibles à la qualité du complément, il est possible que la subdivision entre BGK et $\mathrm{BGKO}^{\prime \prime}$ soit erronée. Notons cependant qu'en race Bringée de Bohême, MATOUŠEK et al. (Ig6I) décrivent également deux allèles distincts qui sont, avec leurs réactifs, $\mathrm{BGK}$ et $\mathrm{BGKO}^{\prime}$.

En définitive, 1'importance de ces erreurs est certainement faible : dans l'ensemble beaucoup de phénogroupes décrits correspondent visiblement à des phénogroupes cités par ailleurs, notamment en race Simmental (MÜLLER, I96I). D'autre part, les principaux phénogroupes "Montbéliard" absents en Simmental ont été vérifiés au laboratoire de Copenhague. Si, par suite de quelques erreurs, le nombre d'allèles de la race a pu être surestimé, à l'inverse, l'utilisation d'anticorps supplémentaires doit permettre ultérieurement de nouvelles dichotomies. Certaines sont suggérées par les quelques tests effectués avec anti-P, anti- $\mathrm{D}^{\prime}$ et anti- $\mathrm{Y}^{\prime}$ : ainsi une partie seulement des phénogroupes $\mathrm{BO}_{3} \mathrm{Y}_{2}$, I et $\mathrm{Y}_{1} \mathrm{I}^{\prime}$ comportent aussi respectivement. $D^{\prime}, P$ ou $Y^{\prime}$ (tableau 9). 


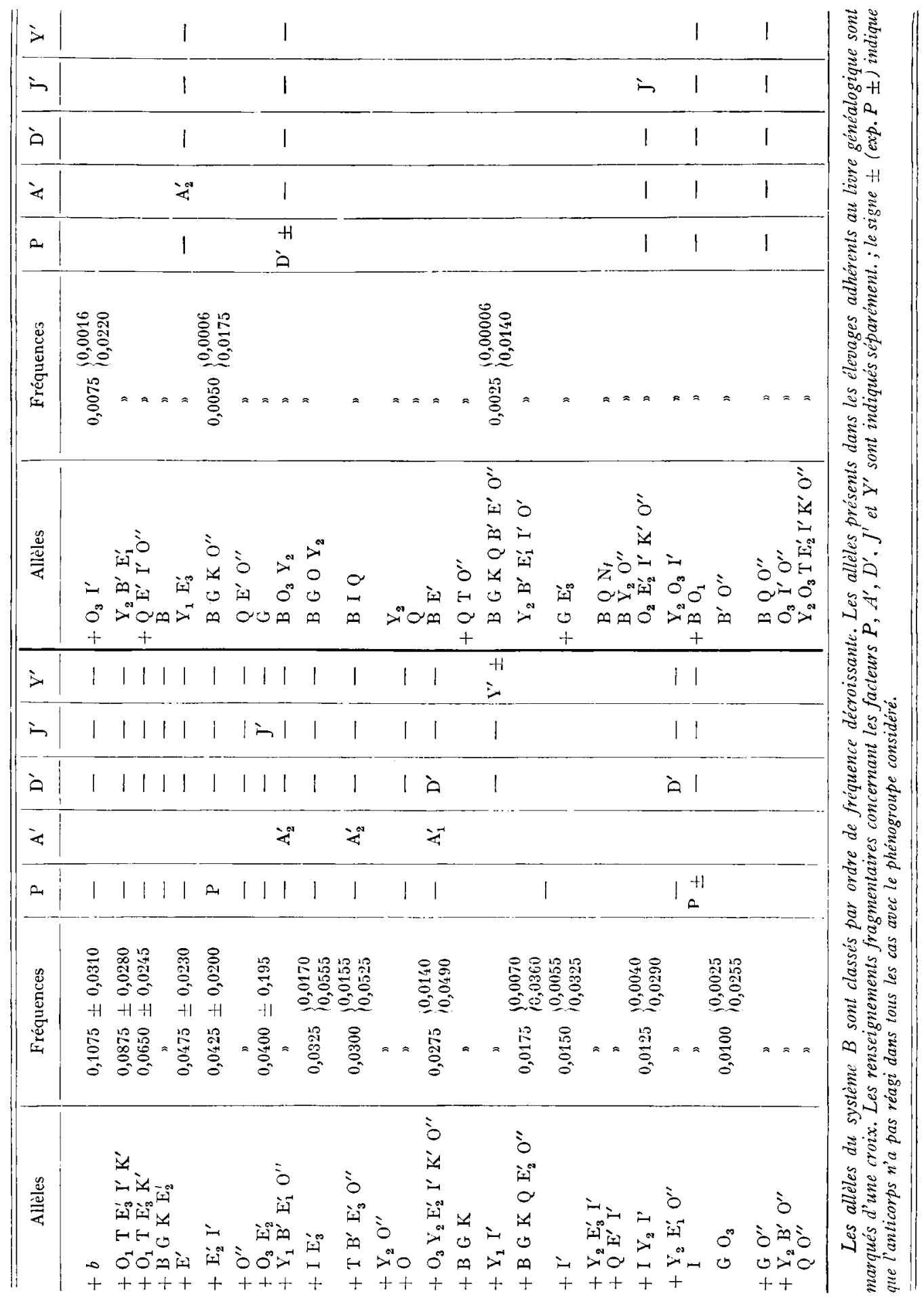




\section{b) Comparaison des élevages affiliés et non affliés au Livre Généalogique de la race}

75 mères seulement sur 400 sont inscrites au Livre Généalogique. Néanmoins, 22 des 23 allèles dont la fréquence dépasse o,or sont représentés dans leur groupe (indiqués : + sur le tableau 9). Il n'y a donc pas de différence qualitative entre les allèles présents dans les élevages adhérents ou non adhérents au Livre Généalogique de la race.

\section{c) Influence des premières années d'insémination artificielle sur les fréquences géniques}

Le Centre d'insémination artificielle du département du Doubs s'est créé en I948. Les groupes sanguins des premiers taureaux mis en service et éliminés depuis n'ont pas été déterminés; cependant nous avons pu retrouver aisément, a posteriori, la plupart des allèles qu'ils possédaient et qui ont été multipliés dans la population par l'insémination artificielle.

En effet ces taureaux sont pères d'une partie des vaches figurant dans l'échantillon. Considérons un tel taureau, de génotype $B_{\alpha} / B_{\beta}$ au locus $B$, et un groupe de ses filles et soient $\mathrm{B}_{1}, \mathrm{~B}_{2} . ., \mathrm{B}_{\alpha}, \mathrm{B}_{\beta} . ., \mathrm{B}_{\imath}$, les allèles de la population, de fréquence respective $f_{1}, f_{2} . ., f_{\alpha}, f_{\beta} . ., f_{i}$; la fréquence des allèles transmis par les filles de ce taureau à leurs produits sera :

$$
\text { pour } \mathrm{B}_{\alpha}: \frac{\mathrm{I}+f_{\alpha}}{4} \text {, pour } \mathrm{B}_{\beta}: \frac{\mathrm{I}+\hat{f}_{\hat{\kappa}}}{4} \text {, et pour } \mathrm{B}_{i}: \frac{f_{i}}{4}
$$

Comme dans la race on a : maximum de $f_{i}<0$, II, les fréquences de $\mathrm{B}_{\alpha}$ et $\mathrm{B}_{\beta}$ chez les produits seront d'environ 0,25 et 0,25 (ou 0,50 pour un seul allèle, si le taureau était homozygote) contre 0,03 au maximum pour 1'un quelconque des $f_{i}$; il suffira donc d'un nombre de couples fille-produit assez restreint pour déterminer les allèles du taureau; en outre, on pourra vérifier que l'un au moins des allèles ainsi trouvés est présent chez chacune des filles.

Les génotypes suivants ont été reconstitués :

TABLEAU IO

Génotypes au locus $B$ des premiers taureaux utilisés pour l'insémination artificielle dans le débarlement du Doubs

\begin{tabular}{|c|c|c|}
\hline Taureaux & Nombre total d'inséminations & Génotype \\
\hline Lutteur $9887 \ldots \ldots \ldots \ldots \ldots \ldots$ & 28300 & $\mathrm{O}_{3} \mathrm{Y}_{2} \mathrm{D}^{\prime} \mathrm{E}_{2}^{\prime} \mathrm{I}^{\prime} \mathrm{K}^{\prime} \mathrm{O}^{\prime \prime} / \mathrm{O}_{1} \mathrm{TE}_{3}^{\prime} \mathrm{K}^{\prime}$ \\
\hline Orphéon $12464 \ldots \ldots \ldots \ldots \ldots$ & 19000 & $\mathrm{Y}_{1} \mathrm{~B}^{\prime} \mathrm{E}_{1}^{\prime} \mathrm{O}^{\prime \prime} / \mathrm{O}_{3} \mathrm{E}_{2}^{\prime} \mathrm{J}^{\prime}$ \\
\hline Bambi $12314 \ldots \ldots \ldots \ldots \ldots$ & 13800 & $\left(\mathrm{Y}_{1} \mathrm{~B}^{\prime} \mathrm{E}_{1}^{\prime} \mathrm{O}^{\prime \prime}\right) / \mathrm{O}_{1} \mathrm{TE}_{3}^{\prime} \mathrm{I}^{\prime} \mathrm{K}^{\prime}$ \\
\hline Mondain $11585 \ldots \ldots \ldots \ldots \ldots$ & 13000 & $\mathrm{BGKE}_{2}^{\prime} / Y_{1} \mathrm{E}_{\mathrm{g}}^{\prime} \mathrm{I}^{\prime}$ \\
\hline Capitaine $12692 \ldots \ldots \ldots \ldots \ldots$ & 12500 & non déterminé \\
\hline IIanneton $8663 \ldots \ldots \ldots \ldots \ldots$ & 10200 & $\mathrm{IE}_{3}^{\prime} /$ non déterminé \\
\hline Prince $12116 \ldots \ldots \ldots \ldots \ldots \ldots$ & 10100 & $Y_{1} I^{\prime} Y^{\prime} / Y_{2} D^{\prime} E_{1}^{\prime} O^{\prime \prime}$ \\
\hline Idéal $9226 \ldots \ldots \ldots \ldots \ldots \ldots$ & 9100 & $\mathrm{O}_{3} \mathrm{E}_{2}^{\prime} \mathrm{J}^{\prime} /$ non déterminé \\
\hline
\end{tabular}


Par ailleurs le taureau Boby, encore vivant, mais père de plusieurs mères dans 1'échantillon est vraisemblablement homozygote pour 1'allèle $\mathrm{O}_{1} \mathrm{TE}_{3}^{\prime} \mathrm{I}^{\prime} \mathrm{K}^{\prime}$.

Dans certains cas, l'effectif des filles connues du taureau s'est avéré trop faible pour déterminer son génotype, ou même un seul de ses allèles avec vraisemblance. Capitaine, par exemple, a été autant utilisé que Mondain, mais il y a eu plus d'éliminations dans sa descendance et son influence génétique est donc plus réduite.

Un groupe de I Ig couples où la mère n'était pas, selon les éleveurs, issue d'insémination, a été extrait de l'échantillon; les fréquences alléliques de ce groupe ont

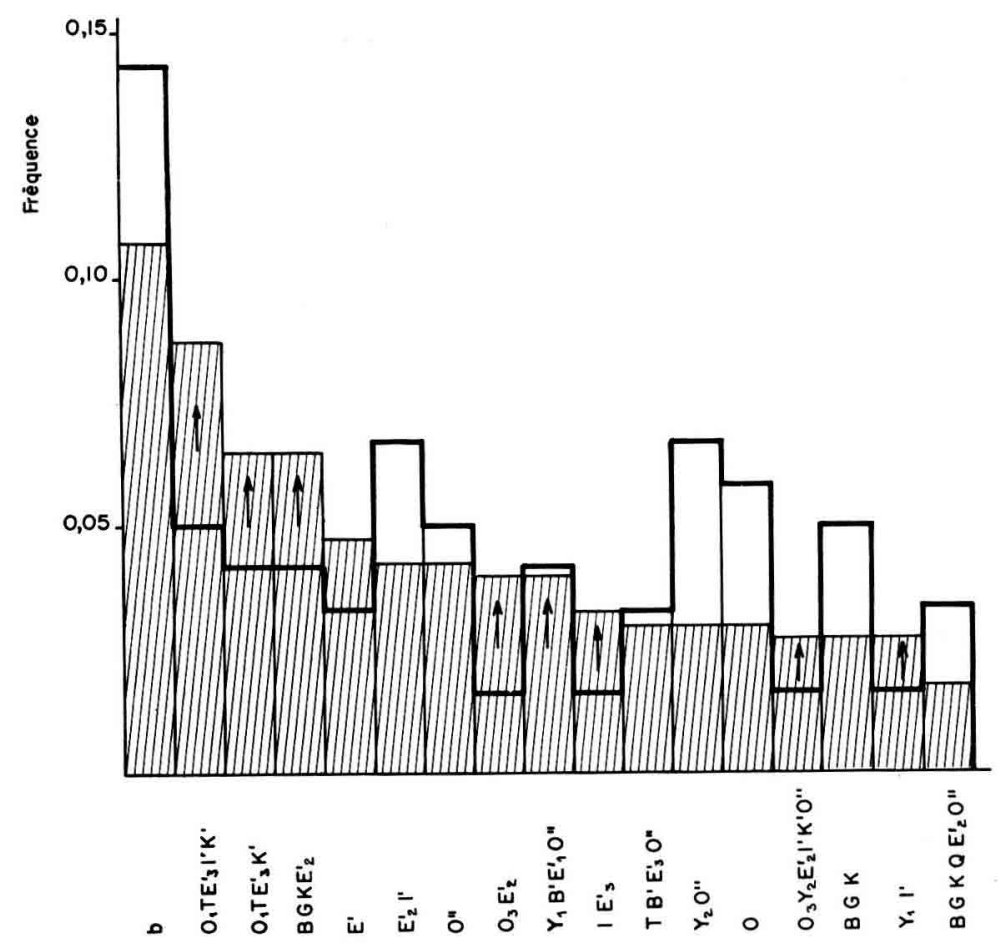

FIG. 2. - Influence de la pratique de l'insémination artificielle sur les fréquences géniques principales : L'histogramme hachuré est celui des fréquences actuelles dans la population (échantillon.) L’histogramme non hachuré superposé est celui des fréquences dans un groupe de I Io vaches non issues d'insémination artificielle. Les flèches indiquent les gènes effectivement multipliés par les taureaux utilisés pour l'insémination artificielle.

été calculées. Elles représentent en principe — mais aux fluctuations d'échantillonnage près, qui sont importantes — les fréquences géniques de la population avant intervention de l'insémination artificielle. La figure 2, qui doit s'interpréter en tenant compte de la faible précision des estimations, fait apparaître assez nettement néanmoins les fluctuations dues à l'insémination artificielle. La fréquence globale des Io phénogroupes que l'on sait multipliés par l'insémination artificielle est de $0,413+0,049$ dans l'échantillon total ; elle est de 0,224 $\pm 0,079$ dans le groupe de I 9 produits des mères non issues d'insémination artificielle. Or, selon le tableau 3 $5^{\circ} \mathrm{p}$. Ioo des mères sont issues d'insémination artificielle : en retenant 0,244 comme fréquence de départ du groupe des gènes précédents et en admettant que les mères 
sont toutes de première génération d'insémination artificielle, la fréquence actuelle attendue est de $0,244+0,5 / 2=0,494$. Compte tenu des erreurs d'échantillonnage, cette valeur concorde assez bien avec la valeur observée de $0,4 \mathrm{I} 3 \pm 0,049$.

Enfin on remarque que l'augmentation relative des fréquences géniques n'est pas égale pour les différents gènes multipliés. Un cas typique est celui de Lutteur ; son utilisation a relativement moins augmenté la fréquence de $\mathrm{O}_{\mathbf{1}} \mathrm{TE}_{3}^{\prime} \mathrm{K}^{\prime}$ que celle de $\mathrm{O}_{3} \mathrm{Y}_{2} \mathrm{D}^{\prime} \mathrm{E}_{2}^{\prime} \mathrm{I}^{\prime} \mathrm{K}^{\prime} \mathrm{O}^{\prime \prime}$ qui était beaucoup plus rare, auparavant, dans la population; ainsi 8 phénogroupes $\mathrm{O}_{3} \mathrm{Y}_{2} \mathrm{D}^{\prime} \mathrm{E}_{2}^{\prime} \mathrm{I}^{\prime} \mathrm{K}^{\prime} \mathrm{O}^{\prime \prime}$ sur II recensés dans l'échantillon, proviennent de ce taureau. De même, plus de la moitié des phénogroupes $\mathrm{Y}_{1} I^{\prime}\left(\mathrm{Y}^{\prime}\right)$ et $\mathrm{Y}_{1} \mathrm{~B}^{\prime} \mathrm{E}_{1}^{\prime} \mathrm{O}^{\prime \prime}$ proviennent respectivement de Prince et Idéal $9 \mathrm{I} 28$ (par lui-même en saillie naturelle, ou par ses fils Mondain et Bambi).

\section{d) Observations sur les génotypes des mères}

L'influence sur les fréquences géniques de la limitation du nombre de reproducteurs, conséquence de l'utilisation de l'insémination artificielle, étant nette, il était intéressant de vérifier si elle pouvait se mesurer au niveau des génotypes des mères figurant dans l'échantillon.

Il aurait été intéressant đe calculer les estimations des fréquences géniques par la méthode de NEIMANN-SORENSEN (I956) pour les comparer aux estimations obtenues par test-cross. Malheureusement nous avons dûu renoncer à analyser en détail les résultats obtenus sur les mères, les analyses n'ayant pas toutes été effectuées, comme pour les produits, avec nos nouveaux réactifs anti-I' et anti- $\mathrm{O}_{3}$.

Néanmoins quelques observations restent possibles : ainsi la fréquence de l'allèle négatif étant $0,1075 \pm 0,0310$, les limites de l'intervalle de confiance à 5 p. Ioo pour le nombre des mères homozygotes dans 1'hypothèse de panmixie, sont 2 et 7 ; le nombre observé est de I ; la déviation est significative ; 1'hypothèse serait donc infirmée.

D'autre part, on peut tester l'hypothèse d'équilibre génétique pour certains groupes d'allèles directement reconnaissables dans les phénotypes; c'est le cas ici pour les allèles comportant BGK ou OTE' $K^{\prime}$.

Dans le cas des allèles comportant BGK (tableau II), l'hypothèse n'est pas infirmée

TABLEAU II

Test de l'hypothèse de panmixie au locus $B$ pour les alleles comportant $B G K$; $q$ désigne la fréquence du group: d'allèles ne comportant pas $B G K$

\begin{tabular}{l|c|c|c}
\hline \hline Phénotype des mères & comportant BGK & sans BGK \\
\hline Phénotype des produits ... & avec BGK & sans BGK & sans BGK \\
\hline Nombre théorique ....... & $\mathrm{N}(1-q)=47$ & $\mathrm{Npq}=41,5$ & $\mathrm{Nq}^{2}=311,5$ \\
\hline Nombre observé......... & 47 & $\mathbf{4 5}$ & 318 \\
\hline
\end{tabular}

$\left(\chi_{2}=I, I 5\right)$. Pour les allèles comportant $\mathrm{OTE}^{\prime} \mathrm{K}^{\prime}$, les chiffres obtenus sont les suivants :

Nombre théorique : $62 \quad 52,4 \quad 285,6$

Nombres observés : $62 \quad 52 \quad 286$

Ici également 1'hypothèse de panmixie n'est pas infirmée $\left(\chi_{{ }_{2}}=0,004\right)$. 
On peut aussi s'intéresser aux phénotypes qui correspondent aux génotypes homozygotes pour les allèles principaux. Le tableau suivant donne les valeurs obtenues pour les phénotypes correspondant aux I I allèles les plus fréquents après (b) (en tenant compte du fait que I' n'a pas été utilisé chez les mères).

TABI,EAU I2

I'st de l'hypothese d"équilibre génitique au locus $B$

$\begin{gathered}\text { Phénotypes } \\ -\begin{array}{c}\text { Nombre théorique } \\ \text { (arrondi) }\end{array}\end{gathered} \mid$ Nombre observé $\mid \chi_{2}$ (1 degré de liberté)

(1) Phénotype correspondant à des allèles multipliés par les taureaux d'insémination artificielle.

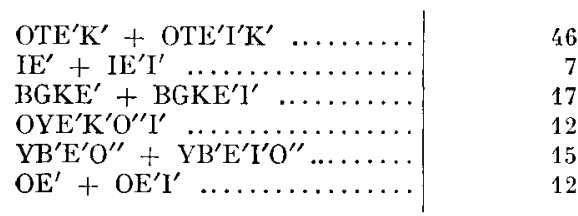

\begin{tabular}{r|r|r}
46 & 49 & 0,20 \\
7 & 12 & 3,57 \\
17 & 6 & 7,12 \\
12 & 9 & 0,75 \\
15 & 17 & 0,27 \\
12 & 15 & 0,75
\end{tabular}

(2) Phénotypes correspondant aux autres allèles.

\begin{tabular}{|c|c|c|c|}
\hline $\mathrm{TB}^{\prime} \mathrm{E}^{\prime} \mathrm{O}^{\prime \prime}+\mathrm{TH}^{\prime} \mathrm{E}^{\prime} \mathrm{I}^{\prime} \mathrm{O}^{\prime \prime} \ldots \ldots \ldots$ & 7 & 5 & 0,57 \\
\hline $\mathrm{YO}^{\prime \prime}+\mathrm{YI}^{\prime} \mathrm{O}^{\prime \prime} \ldots \ldots \ldots$ & 6 & 10 & 2,67 \\
\hline$E^{\prime}+E^{\prime} I^{\prime} \ldots \ldots$ & 12 & 6 & 3,00 \\
\hline $\mathrm{O}^{\prime \prime}+\mathrm{I}^{\prime} \mathrm{O}^{\prime \prime} \ldots \ldots \ldots \ldots \ldots \ldots \ldots$ & 5 & 6 & 0,20 \\
\hline $\mathrm{O}+\mathrm{OI}^{\prime} \ldots \ldots \ldots \ldots \ldots \ldots \ldots$ & 4 & 6 & \\
\hline
\end{tabular}

La seule déviation importante est celle du phénogroupe (BGKE $\left.{ }^{\prime}+\mathrm{BGKE}^{\prime} \mathrm{I}^{\prime}\right)$.

En réalité, ces phénogroupes représentent non seulement les homozygotes mais aussi des hétérozygotes de 2 types : d'une part ceux où le phénogroupe considéré masque un autre phénogroupe (par exemple pour BGKE', E' peut être masqué par BGKE') et d'autre part ceux où l'accolement de deux phénogroupes moins complexes donne le phénotype considéré (par exemple BGK $\div \mathrm{E}^{\prime}$ ), que nous appellerons phénogroupes complémentaires.

Considérons un seul allèle quelconque, et soit $m$ sa fréquence chez les mères formant une population en équilibre génétique, $p$ sa "fréquence de multiplication " par les pères, taureaux d'insémination artificielle en nombre réduit; la fréquence des homozygotes augmentera dans la génération suivante si $p>m$. Mais on peut vérifier également que si cette condition est remplie, le nombre des hétérozygotes pour cet allèle augmente également si $m<0,5$, condition toujours remplie au locus B.

Mais le nombre de phénotypes représentant entre autres les homozygotes dépend de la valeur de 6 fréquences :

- (I) la fréquence du phénogroupe considéré chez les mères et (2) sa fréquence de multiplication par les pères,

- (3) la fréquence des phénogroupes "masqués " chez les mères et (4) leur fréquence de multiplication par les pères,

- (5) la fréquence chez les mères et (6) la fréquence de multiplication par les pères de phénogroupes "complémentaires " (on remarquera qu'un phénogroupe " com- 
plémentaire " dans certaines combinaisons est forcément masqué par l'allèle considéré).

La figure 3 illustre le cas de l'allèle $B G K K^{\prime}$ (BGKE'I' exclu) pour des fréquences prises arbitrairement :

Les interactions entre les 6 fréquences intéressantes conduisent à masquer l'évolution du nombre des homozygotes. Considérons les valeurs réellement obtenues dans le cas de $\mathrm{BGKE}^{\prime}+\mathrm{BGKE}^{\prime} \mathrm{I}^{\prime}$. La fréquence de multiplication de $\mathrm{BGKE}^{\prime}$ par le groupe des taureaux est, d'après le nombre des inséminations, voisine de $5,6 \mathrm{p}$. Ioo - sans doute un peu supérieure — donc voisine de la fréquence antérieure de l'allèle dans la population. Le nombre d'homozygotes doit rester sensiblement stationnaire ainsi que le nombre d'hétérozygotes où figure $B G \mathrm{KE}^{\prime}$ provenant des taureaux. Mais aucun allèle "masqué " ou complémentaire n'est transmis par le groupe des taureaux done le nombre total de phénotypes BGKE' doit diminuer, ce qui est

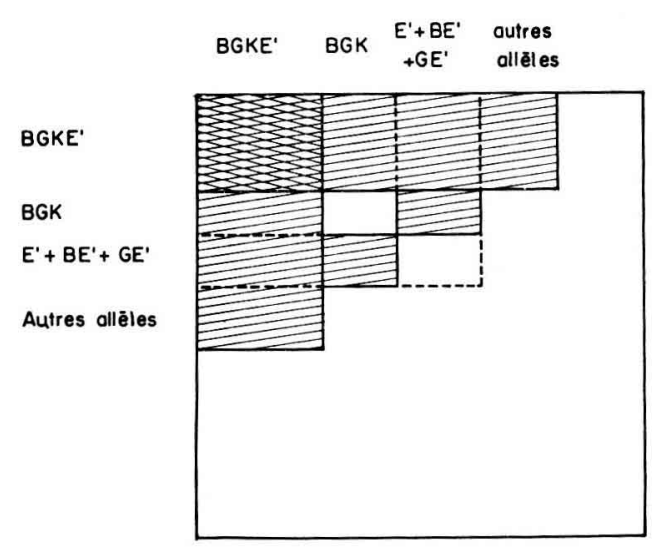

FIG. 3. - Illustration des interactions alléliques intervenant dans la tormation d'un même phénotype: sur les lignes et les colonnes, segments proportionnels aux fréquences alléliques chez les parents (valeurs arbitraires). Les surfaces hachurées sont proportionnelles à la tréquence du phénotype $B G K E$ ' che les produils.

effectivement le cas. Néanmoins la situation est très voisine pour l'allèle $\mathrm{IE}_{3}^{\prime}$ où le nombre de phénotypes $\mathrm{IE}_{3}^{\prime}\left(\mathrm{I}^{\prime}\right)$ a pourtant augmenté. En fait les fluctuations d'échantillonnage ont une influence importante.

En conclusion, même en connaissant avec sûreté les phénotypes maternels, les observations qui peuvent être faites sur eux sont limitées par les fluctuations d'échantillonnage et surtout par le fait qu'on ne puisse pas distinguer les génotypes homozygotes des génotypes hétérozygotes qui conduisent au même phénotype.

\section{DISCUSSION}

NeIMANN-Sorensen (I958), utilisant I5 réactifs pour le système B, décrivait 24 allèles en race Rouge Danoise (R. D. M), 2 I en race Frisonne Danoise (S. D. M) et ${ }_{5}$ en race de Jersey. Depuis, disposant de nouveaux anticorps et sous-groupes 
d'anticorps, LARSEN (I96I), au même laboratoire, décrit respectivement 55, 63 et 24 allèles dans les races R.D.M., S.D.M. et Jersey. Matousé et al. (Ig6I) ont découvert également un nombre élevé d'allèles - 63 - en race Bringée de Bohême et concluent à une forte variabilité. La présente étude de la race Montbéliarde a été effectuée avec une série d'anticorps comparable plutôt à celle dont disposait NEIMANN-SORENSEN en I958. Il est donc certain que le nombre de 55 allèles trouvé dans la race $M o n t b e ́-$ liarde sous-estimele nombre d'allèles réel, et que ce dernier est élevé. RoBERTSON (I956) propose de caractériser la variabilité d'une race par le " nombre d'allèles utile") " effective number of alleles $"$ défini par $\mathrm{N}_{u}=\mathrm{I} / \Sigma_{i} q_{i}^{2}, q_{i}$ étant une des fréquences alléliques. En $R$. D. $M$., Neimann-Sorensen (I958) avait trouvé $\mathrm{N}_{u}=7$. En race Montbéliarde, on trouve $\mathrm{N}_{u}=22$. Cette race se caractérise donc par une forte variabilité génétique; ses possibilités d'évolution par sélection devraient être encore importantes.

Si l'échantillon analysé donne certainement une image assez fidèle de la population de la zone de sondage, il est vraisemblable qu'il ne représente qu'approximativement la race Montbéliarde dans son ensemble. Nemmans-Sorensen (I958) et RendeI, (I958) ont montré qu'il existait dans les races danoises et suédoises des différences de fréquences géniques entre certains groupes zootechniques ou géographiques, et BRAEND (I959) pose la question de savoir si les fréquences alléliques trouvées dans une zone donnaient une image fidèle de celles de la race. Il est certain que seul un sondage stratifié s'adressant à la population entière en donnerait une image exacte ; ceci est particulièrement vrai en zone montbéliarde où l'ancienneté de l'introduction du type Montbéliard varie selon les régions. En fait, c'est au type Montbéliard dit "pur " que nous avons voulu nous intéresser en choisissant la zone de sondage en bordure du " berceau de la race "; d'ailleurs l'utilisation, dans le département du Doubs, de l'insémination artificielle en dehors du berceau de la race, a dû niveler, en partie, les différences qui pouvaient exister entre diverses zones. L'échantillon analysé donne donc vraisemblablement une image assez exacte du type Montbéliard dans son département d'origine.

La figure 4 met en parallèle les fréquences alléliques au locus $\mathrm{B}$ dans les races Montbéliarde et Tachetée Rouge de Simmental (MÜLLER, I960). Les deux races n'ont pas été étudiées avec tous les mêmes réactifs et dans certains cas la correspondance indiquée n'est que vraisemblable.

On constate que la plupart des allèles de la race Montbéliarde ont leur équivalent probable en race Simmental; néanmoins des différences notables se révèlent :

- 4 allèles parmi les plus fréquents en race Montbéliarde n'ont pas été signalés en race Simmental: $\mathrm{Y}_{1} \mathrm{~B}^{\prime} \mathrm{E}_{1}^{\prime} \mathrm{O}^{\prime \prime}, \mathrm{TB}^{\prime} \mathrm{E}_{3}^{\prime} \mathrm{O}^{\prime \prime}, \mathrm{O}_{3} \mathrm{Y}_{2} \mathrm{D}^{\prime} \mathrm{E}_{2}^{\prime} \mathrm{I}^{\prime} \mathrm{K}^{\prime} \mathrm{O}^{\prime \prime}$ et $\mathrm{Y}_{1}^{\prime} \mathrm{I}^{\prime} \mathrm{Y}^{\prime}$. En fait l'étude de MüL,LER n'a porté également que sur un petit échantillon de la race et on peut s'attendre à ce que certains gènes, plus ou moins rares, n'aient pu alors être mis en évidence. SCHINDLER (Ig62) signale la découverte d'un allèle TA $\mathrm{T}_{1}^{\prime} \mathrm{E}_{3}^{\prime}$ qui pourrait correspondre à $\mathrm{TB}^{\prime} \mathrm{E}_{3}^{\prime} \mathrm{O}^{\prime \prime}$ Montbéliard (allèle qui réagit avec $\mathrm{A}_{2}^{\prime}$ danois). Cette hypothèse devra être vérifiée.

Seul des 4 allèles de la race Montbéliarde, absents en race Simmental, TB' $\mathrm{E}_{3}^{\prime} \mathrm{O}^{\prime \prime}$ n'a pas été multiplié par un taureau d'insémination artificielle; il est cependant réparti dans toute la zone de sondage (carte 2 ) et apparaît comme un des principaux allèles de la race. 
- Par ailleurs, il y a de fortes différences entre les fréquences alléliques des 2 races. Les allèles $\mathrm{OI}^{\prime}$ et $\mathrm{GA}^{\prime}$ qui dominent en race Simmental - le cas particulier de $b$ étant mis à part — sont rares en race Montbéliarde.

Quelques autres observations peuvent être faites : ainsi l'allèle $A Z$ ' du locus $\mathrm{A}$ de fréquence 0, or 7 en race Montbéliarde, n'a pas été trouvé jusqu'à présent en Simmental (SCHINdiER, I962). La présence de cet allèle provient peut-être de l'ancien bétail local. A cet égard, le facteur $Z^{\prime}$, rare ou absent dans les races d'origine hollandaise, anglaise et scandinave, est plus courant en France, et sa fréquence atteint même 28 p. Ioo environ en race Charolaise.

Par ailleurs c'est peut-être dans le département de la Saône-et-Loire que subsistent quelques réminiscences des anciennes races locales comme la Fémeline. Un sondage réduit effectué en Ig60 dans la région de Verdun-sur-le-Doubs, semble

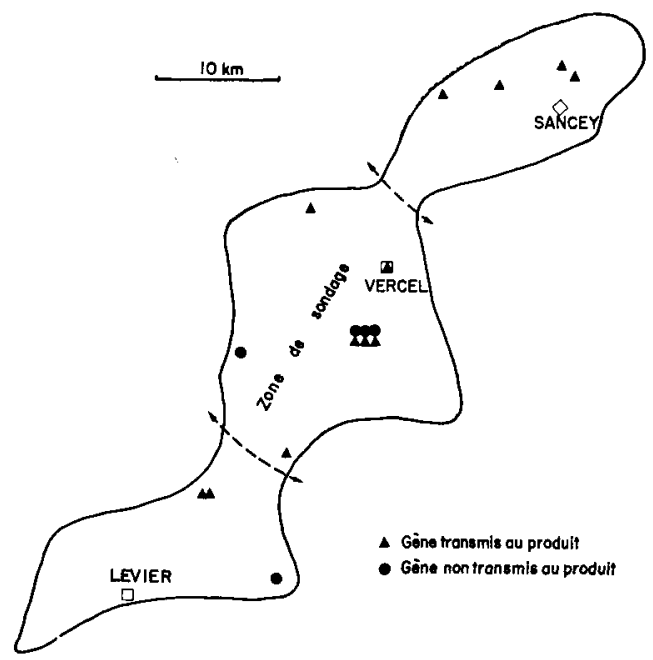

CARTE 2 - Localisation, dans la zone de sondage, et pour l'ćchantillon, du gène 'TB' $\mathrm{E}_{3}^{\prime} \mathrm{O}^{\prime \prime}$, non signalé jusqu'ł présent en race Simmental et non multiplié par l'insémination artificielle. On distingue les cas où le gène a été transmis au produit et ceux où il est seulement reconnaissable chez les mères. Dans un village au sud de Vercel, un taureau utilisé dans plusieurs étabjles possćdait ce gène.

indiquer des différences de fréquence allélique notables avec le sondage de la zone du Doubs. Ainsi $\mathrm{O}_{1} \mathrm{TE}_{3}^{\prime} \mathrm{K}^{\prime}$ et $\mathrm{O}_{1} \mathrm{TE}_{3}^{\prime} \mathrm{I}^{\prime} \mathrm{K}^{\prime}$ y indiquent souvent une immigration d'animaux venant du Doubs, alors que parmi les allèles fréquents figure QTO' (vraisemblablement $\mathrm{A}^{\prime}$ ) non signalé en Simmental.

Des sondages locaux présenteraient un grand intérêt. A cet égard il est remarquable que 3 allèles non signalés en Simmental aient été présents chez les premiers taureaux utilisés par le Centre d'insémination du Doubs. I1 se peut que l'originalité des groupes sanguins de la race Montbéliarde soit encore plus accusée dans son berceau de race.

De toute façon, on doit tenir compte dans cette comparaison raciale, du fait que les races d'Europe centrale, telles que la Simmental (MúlLER, I96o), la Fribourgeoise (SCHINDLER, Ị66I), la race Bringée de Bohême (MATOUŠEK et al., I96I), la race Rouge Polonaise (NeIMANN-SORENSEN et SPRYSzAK, 1959), etc., ont certains liens de parenté et possèdent un nombre plus ou moins grand d'allèles en commun. 
Le bétail local sur lequel s'est implantée la race Bernoise lors de la création de la race Montbéliarde (VERNIER, I953) n'était sans doute pas totalement différent des apports qu'il reçut.

Roce Montbaliorde

Roce Simmentol

(Mülter 1960)

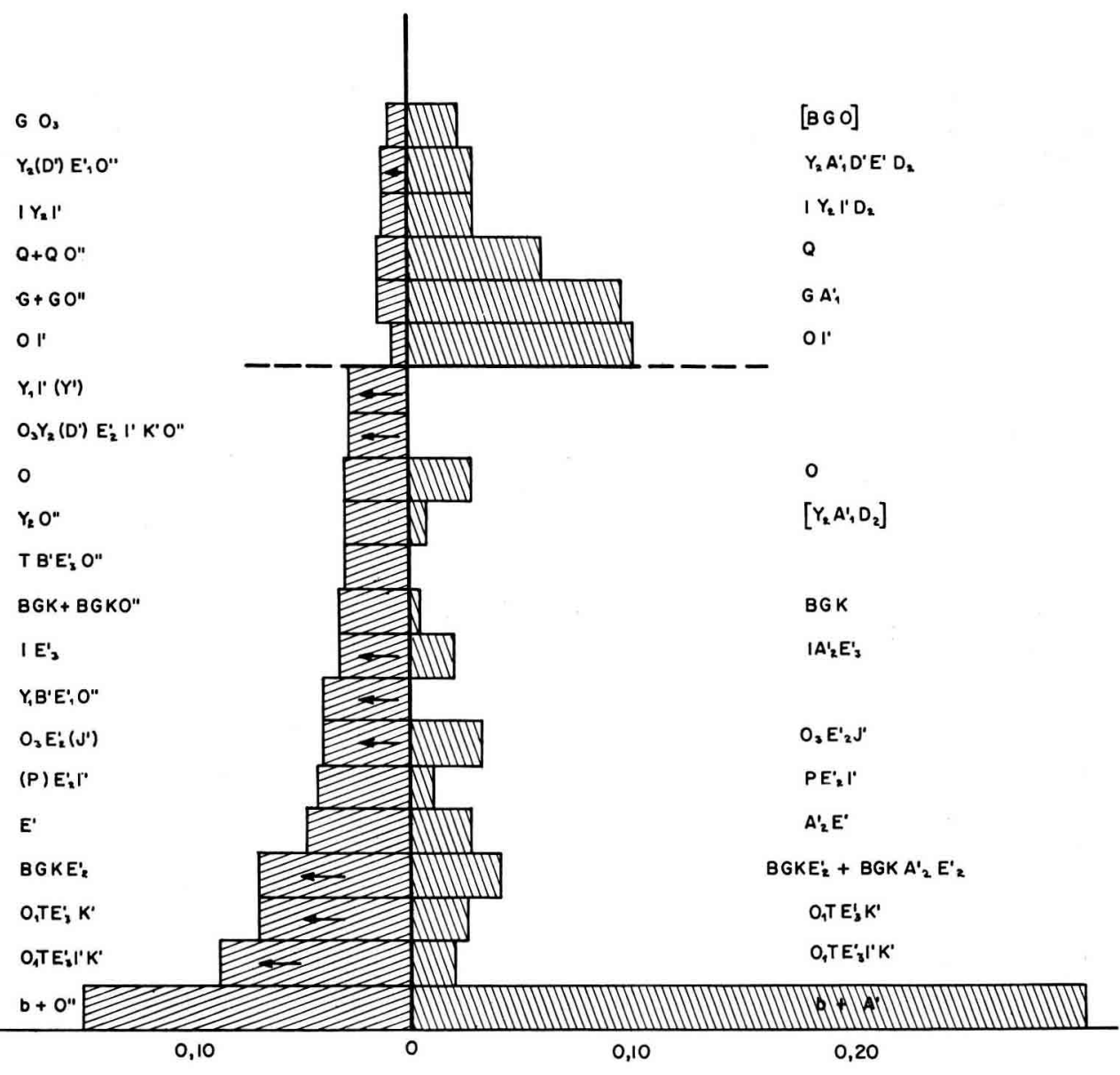

Fréquences

Fig. 1.- - Comparaison qénétique entre les races Montbéliarde et Tachetée du Simmental Suisse pour les fréquences alléliques qui sont supérieures à 0.02 dans l'une des deux races au moins. Les allèles de frénuence 0,02 sont classés par ordre de fréquence décroissante dans la race Montbáliarde d'abord, puis dans la race Simmental. Les deux races n'ayant pas été analysées anec les mêmes récuctits exactement, la correspondance est seulement araisemblable : les crochets indiquent les allèles pour lesquels elle l'est le moins; les parenthèses indiquent les facteurs pour lesquels le réactif correspondlant n'a pas été utilisé dans toutes les analyses.

En conclusion, la parenté des deux races est évidente, mais il est clair également que le processus de leur isolement génétique est nettement engagé.

Il ne fait pas de doute, d'après nos résultats, que la limitation du nombre de reproducteurs mâles, corollaire de l'utilisation de l'insémination artificielle, a eu, même prise à ses débuts - beaucoup de vaches sont de première génération d'insémination artificielle — des répercussions assez accusées pour que la population ne 
puisse pas être considérée comme étant en équilibre génétique. Les déviations à attendre théoriquement sont différentes de celles qui caractérisent un processus d'évolution vers la consanguinité puisque, par exemple, si le nombre des homozygotes doit augmenter (comme les gènes multipliés l'ont été à une fréquence supérieure à la fréquence antérieure dans la race), celui de certains hétérozygotes également. Le fait que les génotypes homozygotes ne puissent être distingués empêche de mettre en évidence les déviations de façon convaincante, au locus B par exemple. L'hypothèse d'équilibre génétique a été vérifiée aux loci $\mathrm{A}$ et SU; elle semble infirmée au locus SU mais non au locus $A$. Il est probable que les génotypes des taureaux utilisés donnaient au total une image suffisamment approchée des fréquences de la population pour que leurs effets individuels se compensent sensiblement. I)ans les travaux étrangers, l'hypothèse d'équilibre génétique n'a pas en général pu être infirmée; néanmoins Bouw (I958, I960) citant le cas de lignées particulièrement utilisées en Hollande, doute que l'équilibre génétique soit vraiment la règle générale.

Une autre voie d'approche de l'analyse de la structure génétique de la population est l'étude de l'évolution des fréquences géniques dans la zone de sondage; les calculs portent ici sur les fréquences elles-mêmes, et non plus sur leurs carrés, donc sur des chiffres plus élevés et plus facilement analysables. Nous avons pu ainsi désigner les gènes qui avaient été multipliés dans la population et apprécier approximativement l'ampleur de cette évolution; des sondages ultérieurs dans la même zone pourront permettre de suivre l'évolution de la population, où la pratique de l'insémination artificielle se généralise rapidement. Deux orientations opposées pourront se manifester : ou bien préférence sera donnée dans le choix des reproducteurs à quelques lignées, ce qui conduira à multiplier fortement certains gènes, ce qui a été le cas en race Shorthorn, au XIX ${ }^{e}$ siècle, pour le gène $\mathrm{BOY}_{1} \mathrm{~A}^{\prime} \mathrm{E}_{3}^{\prime}$ (NEIMANN-SORENSEN, I956 - ROBERTSON, I956). Cette évolution se manifeste actuellement dans le département du Jura où l'allèle $\mathrm{O}_{1} \mathrm{TE}_{3}^{\prime} \mathrm{I}^{\prime} \mathrm{K}^{\prime}$ par exemple, est multiplié par la famille du fameux taureau Océano; ou bien la recherche de souches variées retardera cette concentration.

Le coefficient de parenté de la population, c'est-à-dire la probabilité pour que deux loci homologues de deux individus pris au hasard dans la population, soient occupés par le même gène provenant sans mutation d'un ancêtre commun, a pour borne supérieure $\mathrm{Pa}=\sum_{i} q_{i}^{2}$ dont la valeur est actuellement $4,5 \mathrm{p}$. roo en race Montbéliarde. On pourra suivre l'évolution ultérieure de la structure de la population par celle de la valeur de $\mathrm{Pa}$.

Il serait intéressant de savoir, le plus souvent possible, avec quelle fréquence un allèle donné provient d'un ancêtre donné. Dans le cas de Lutteur, par exemple, plus de la moitié sinon les $2 / 3$ des animaux possédant $\mathrm{O}_{3} \mathrm{Y}_{2} \mathrm{D}^{\prime} \mathrm{E}_{2}^{\prime} \mathrm{I}^{\prime} \mathrm{K}^{\prime} \mathrm{O}^{\prime \prime}$ sont ses descendants. Comme ce taureau était améliorateur, il se peut que ses descendants soient plus souvent conservés que ceux des autres animaux non parents possédant l'allèle ci-dessus ; il y a donc quelques chances pour que, de plus en plus, la présence de cet allèle permette de mesurer le coefficient de parenté moyen de la population vis-à-vis de ce taureau.

Enfin les travaux de RENDEL, (I959, I96I) et de NEIMANN-SORENSEN et ROBERTSON (I96I) ont montré que dans les races S. L. B., S. R. B. et R. D. M., l'allèle $\mathrm{BO}_{1} \mathrm{YD}^{\prime}$ était associé à un taux butyreux du lait plus élevé, ce qui serait aussi le cas pour l'allèle $\mathrm{GD}^{\prime}$ en race de Jersey ; dans cette dernière race, 1'allèle $\mathrm{O}_{1}{ }^{\top} \mathrm{TE}_{3}^{\prime} \mathrm{K}^{\prime}$ serait associé 
à un taux butyreux plus faible (les deux résultats obtenus en race Jersey sont cependant moins bien établis que ceux qui concernent l'allèle $\left.\mathrm{BO}_{1} \mathrm{YD}^{\prime}\right)$. En race Montbéliarde $\mathrm{BOY}_{2} \mathrm{D}^{\prime}$ est rare, $\mathrm{O}_{1} \mathrm{TE}_{3}^{\prime} \mathrm{K}^{\prime}$ fréquent et le taux butyreux moyen est relativement faible, voisin de $37 \mathrm{p}$. 1oo, ou même dans certaines régions de $36,5 \mathrm{p}$. roo (AURIOL, et JARrige, I 662 ). Une recherche sur ce thème présente donc a priori un certain intérêt dans la race Montbéliarde.

\section{CONCI,USION}

La méthode de détermination des allèles par test-cross, choisie pour ce travail, a pu être appliquée avec succès, grâce à la réunion de deux conditions favorables : l'existence d'un taureau posséđant un grand nombre de descendants, et la bonne connaissance des filiations de leur élevage que possèdent en général les éleveurs de la zone prospectée. Il a donc été possible d'établir rapidement une première image des groupes sanguins dans une zone limitée. Ces renseignements devront être complétés ultérieurement grâce à l'utilisation de réactifs supplémentaires; on doit s'attendre également à la mise en évidence d'autres allèles.

Dès à présent, ce travail a permis de définir un "état initial " de la structure génétique de la population dans la zone de sondage où la pratique de l'insémination artificielle se généralise; l'évolution de cette population pourra y être contrôlée à l'avenir. Naturellement ce travail devra être complété par l'étude des groupes sanguins de la race dans une aire plus vaste et en particulier dans le berceau de race où l'insémination artificielle n'est pas pratiquée ; l'étude comparée de l'évolution génétique dans la zone de sondage et dans le berceau de race sera aussi pleine d'intérêt.

$$
\text { Reçu pour publication en juillet } 1962 .
$$

\section{REMERCIEMENTS}

Nous remercions sincèrement le Dr Y. BovQueT, directeur du laboratoire de Gand (Belgique), le professeur $\mathrm{D}^{\mathrm{r}}$ Meyn du Iaboratoire de Munich (Allemagne) et le professeur $\mathrm{D}^{\mathrm{r}} \mathrm{J}$. MoustgaARd du Laboratoire de Copenhague (Danemark) pour leurs dons de réactifs et pour l'aide apportée en analysant certains échantillons.

La réalisation de cette étude a été largemənt facilitée par l'aide technique des organismes d'élevage locaux : nous remercions le Herd-Bonk Montbéliard pour les nombreux services rendus, ainsi que la direction et le personnel des Centres d'Insémination artificielle de Verdun-sur-le-Doubs (Saône-et-Loire) et de Besançon (Doubs), en particulier M. M. LIME, inséminateur à Vercel qui a beaucoup aidé au démarrage de cette étude sur le terrain.

\section{SUMMARY}

BLOOD GROUP STUDIES ON CATTLE OF FRENCH MONTBELIARD BREED

Blood groups of 400 progeny of an artificial insemination bull homozygous for the negative allele at the $\mathrm{B}, \mathrm{J}$ and $\mathrm{Z}$ loci, heterozygous with one negative allele at the $\mathrm{A}$, SU and $\mathrm{L}$ loci, as well as those of their mothers, have been determined in the Montbeliard breed, related to the Swiss Red Pied Simmental breed. 
- With a battery of reagents to be completed, 55 alleles have been found in the B system ; the most common alleles are - not counting allele $b-: \mathrm{O}_{1} \mathrm{TE}_{3}^{\prime} \mathrm{I}^{\prime} \mathrm{K}^{\prime}, \mathrm{O}_{1} \mathrm{TE}_{3}^{\prime} \mathrm{K}$ and $\mathrm{BGKE}_{2}^{\prime} ; p_{i}$ being the frequency of one of them, $\Sigma_{l} p_{i}^{2}=0,045$. These results indicate a large genetic variability.

- Most of these alleles appear to be identical to those described by MúlLer (1960) in the Simmental breed. However certain alleles of the B system, quite frequent in the Montbeliard breed and the allele $A Z^{\prime}$ of the A system, have not been found in Switzerland. Moreover large differences exist between other allelic frequencies. The process of genetic isolation of the two breeds then appears to be engaged.

- The assumption of genetic equilibrium has been tested with regard to the $\mathrm{A}$ and SU loci. There was a significant deviation at the SU locus, but not at the A locus. However, in the B system, shifts due to artificial insemination are shown to be not negligible. Future surveys will permit the control of genetical evolution of the population.

\section{RÉFÉRENCES BIBLIOGRAPHIQUES}

Auriol P., Jarrige R., 1962. Possibilités de modifier la composition du lait. Bull. Feed. Int. Lait. (sous presse)

Bouw J., 1958. Blood group studies in dulch cattle breeds. Thèse, Utrecht, Veenman $\epsilon$ Z Zonen, Wageningen, $84 \mathrm{pp}$.

Bouw J., 1960. The genetical composition of the dutch cattle breeds as determined by the frequencies of blood groups. Z. Tierz. Züicht. Biol, 74, 248-266.

BRAEND M., I959. Blood groups of catle in Noriar - Serological and genetical studies. Skandinavisk Bladforlag. I 44 pp.

Ceppelini R., Siniscalco M., Smith C. A. B., 1956. The estimation of gene frequencies in a random mating population. Ann. IHum. Gen., 20, 97-II5.

FERguson L. C., I94I. Heritable antigens in the erythrocytes of cattle. J. Immum., 40, 213-242.

Ferguson L. C., Stormont C., Irwin M. R., I942. On additional antigens in the erythrocytes of cattle. l. Immum., 44, I47-164.

LARSEN B., 196r. Additional blood-group factors on the A and B systems of cattle. Acta Agric. Scard., 11, $242-256$

Matoušek J., Čuta I., Šrefl J., r 96 I. Alleles of the B, C, FV, M and SU blood group systems of Bohemian brindled cattle. Folia Biol., $, 7,390-394$

MülLER E., 1960. Contribution à l'étude des groupes sanguins de la race Tachetée Rouge de Simmental. Z. Tierz. Zï̌cht. Biol., "74, 89-105

Neimann-Sorensen A., I956. Blood groups and breed structure as exemplified by three danish breeds. Acta Agric. Scand., 6, I I 5-ז 37 .

NeIMANN-SoRensen A., 1958. Blood groups of calle. Immunogenetic situdies on danish calle breeds. A/S Carl Fr. Mortensen, Kobenhavn, i 7 Pp.

Neimann-Sørensen A., Robertson A., I96r. The association between blood groups and several characteristics in three danish cattle breeds. Acta Agric. Scand., 11, 163-196.

Neimann-Sorensen A., Spryszak A., i959. Blood group studies on cattle of Red Polish breed. Anim. Prod., 1, 179-188.

RENDEL J., 1958. Studies of cattle blood groups. IV. The frequency of blood group genes in Sweedish cattle breeds, with special reference to breed structure. Acta Agric. Scand., 8, r91-215.

RENDEL J., 1959. A study on relationship between blood groups and production characters in cattle. VI Cong. Int. Rech. Groupes Sang. Anim., Munich, 8-23.

RENDEL J., 196r. Recent studies on relationships between blood groups and production characters in farm animal. Z. Tiers. Zü̈ht. Biol., 75, 97-109.

RoberTSON A., 1956. Blood grouping in dairy cattle improvement. VII Cong. Int. Zoot., thème $\mathbf{2}$, 79-84.

SCHINDLER A., 196r. Blutgruppenbesiimmungen bei der Freiburger Schwarzfeckvieh Rasse soveie einige praktische Anvendungen der Bluttypiesierung. Art. Institut Orell Füssli A. G. Zürich.

Schinder A., I962. (Communication personnelle.)

Stormont C., 1950. Additional gene-controlled antigenic factors in the bovine erythrocytes. Genetics, 35, 76-94.

StoRMont C., ig6r. Further expansion of the A system of bovine blood groups. Fed. Proc., 20, 66 (abstract) Stormont C., Miller W. J., SuzUki Y., I $96 \mathrm{I}$. The S system of bovine blood groups. Genetics, 46, 541-55I.

Vernifr M., i953. Historique de la race de Montbóliard. Ed. Camponovo, Besançon, 8o pp. 